Cincinnati preprint July.1994

\title{
Renormalizability and Quantum Stability of the Phase Transition in Rigid String Coupled to Kalb-Ramond Fields I
}

\author{
M. AWADA* \\ Physics Department \\ University of Cincinnati,Cincinnati, OH-45221
}

\begin{abstract}
Recently we have shown that a phase transition occurs in the leading approximation of the large $\mathrm{N}$ limit in rigid strings coupled to long range Kalb-Ramond interactions. The disordered phase is essentially the Nambu-Goto-Polyakov string theory while The ordered phase is a new theory. In this part I letter we study the first sub-leading quantum corrections of the free rigid string and derive the renormalization group equation. We show that the theory is asymptotically free, thus the extrinsic curvature of the string drops out at large distance scales in the disordered phase. In part II we generalize the results of this letter to the interacting theory of rigid strings with the long range Kalb-Ramond interactions. We derive the renormalized mass gap equation and obtain the renormalized critical line. Our main and final result is that the phase transition does indeed survive quantum fluctuations.
\end{abstract}

* E-Mail address: moustafa@physunc.phy.uc.edu 
The purpose of this letter is two fold one is to study the free rigid string [1] to the first sub-leading quantum corrections and show that the beta function remains negative, thus implying that the extrinsic curvature becomes irrelevant at large distance scales in the disordered phase. This is of utmost importance for the decoupling of ghosts of the quantum theory [2]. Next the results here will serve as the foundations for solving a more complicated problem. This the problem of showing that the phase transition which we proved [3] to occur in the leading approximation of large $\mathrm{N}$ limit in the theory of rigid strings coupled to the long range Kalb-Ramond interactions, is stable when we include the sub-leading quantum loop corrections in Large N. This would be the topic of part II of this letter.

The gauge fixed action of the free rigid string [1] is :

$$
I_{\text {gauge-fixed }}=\mu_{0} \int d^{2} \xi \rho+\frac{1}{2 t_{0}} \int d^{2} \xi\left[\rho^{-1}\left(\partial^{2} x\right)^{2}+\lambda^{a b}\left(\partial_{a} x \partial_{b} x-\rho \delta_{a b}\right)\right]
$$

where $\mathrm{t}$ is the curvature coupling constant which is dimensionless. The partition function is

$$
Z=\int D \lambda D \rho D x \exp \left(-S_{\text {gauge-fixed }}\right)
$$

The effective action is obtained by integrating over $x^{\nu}, \nu=1, \ldots D$ we have:

$$
S_{0 e f f}=\frac{1}{2 t_{0}}\left[\int d^{2} \xi\left(\lambda^{a b}\left(-\rho \delta_{a b}\right)+2 t_{0} \mu_{0} \rho\right)+t_{0} D \operatorname{tr} \ln A\right]
$$

where $\mathrm{A}$ is the operator

$$
A=\partial^{2} \rho^{-1} \partial^{2}-\partial_{a} \lambda^{a b} \partial_{b}
$$

In the large $\mathrm{D}$ limit the stationary point equations resulting from varying $\lambda$ and $\rho$ respectively are:

$$
\begin{gathered}
\rho=\frac{t_{0} D}{2} \operatorname{tr} G \\
2 t_{0} \mu_{0}-\lambda^{a b} \delta_{a b}=t_{0} D \operatorname{tr}\left(\rho^{-2}\left(-\partial^{2} G\right)\right)
\end{gathered}
$$

where the world sheet Green's function is defined by:

$$
G\left(\xi, \xi^{\prime}\right)=<\xi\left|\left(-\partial^{2}\right) A^{-1}\right| \xi^{\prime}>
$$


The stationary points are:

$$
\rho(\xi)=\rho^{*}, \quad \lambda^{a b}=\lambda^{*} \delta^{a b}
$$

where $\rho^{*}$ and $\lambda^{*}$ are constants. Thus eq.(5a) becomes the mass gap equation:

$$
1=\frac{D t_{0}}{2} \int \frac{d^{2} p}{(2 \pi)^{2}} \frac{1}{p^{2}+m_{0}^{2}}
$$

where we define the mass

$$
m_{0}^{2}=\rho^{*} \lambda^{*}
$$

this yields the mass gap equation,

$$
m_{0}=\Lambda e^{-\frac{4 \pi}{D t_{0}}}
$$

where $\Lambda=\frac{1}{a}$ is an U.V. cut-off and $m_{0}$ is now the bare mass associated with the propagator:

$$
<\partial_{a} x^{\mu}(p) \partial_{a} x^{\nu}(-p)>=\frac{D t_{0}}{2} \frac{\delta^{\mu \nu}}{p^{2}+m_{0}^{2}}
$$

On the other hand eq(5b) yields the string tension renormalization condition:

$$
\mu_{0}=\frac{D}{8 \pi} \frac{\Lambda^{2}}{\rho^{*}}
$$

eq. (10a) agrees exactly with the one loop result.

\section{II-The Loop Corrected Gap Equation and the RG Equation}

In mean field theory i.e leading order in $\frac{1}{D}$, the relevant propagator is equation (10 b). In the sub-leading correction to mean field theory, the quantum fluctuation imply a new term corresponding to the self-energy of the $\partial_{a} x^{\mu}$-field

$$
<\partial_{a} x^{\mu}(p) \partial_{a} x^{\nu}(-p)>=\frac{D t_{0}}{2} \frac{\delta^{\mu \nu}}{\left(p^{2}+m_{0}^{2}+\frac{1}{D} \Sigma(p)\right)} .
$$

The new contribution $\Sigma(p)$ arises from fluctuations of the Lagrange multipliers $\lambda_{a b}$ 
and $\rho$ where the fluctuations $\sigma_{a b}$ and $\eta$ are defined by:

$$
\begin{gathered}
\lambda_{a b}=\lambda^{*} \delta_{a b}+i \frac{1}{\sqrt{(D / 2)}} \sigma_{a b} \\
\rho=\rho^{*}\left(1+i \frac{\rho^{*}}{\sqrt{(D / 2)}} \eta\right) .
\end{gathered}
$$

Expanding the effective action (3) in powers of $\sigma_{a b}$ and $\eta$, it is straightforward to extract the $\sigma_{a b}$ and $\eta$ propagators (Fig (1)):

$$
\begin{gathered}
\Pi_{a b \mid c d}(p)=\frac{1}{2}\left(\delta_{a c} \delta_{b d}+\delta_{a d} \delta_{b c}\right) \pi\left(p^{2}\right) \\
\pi\left(p^{2}\right)=\int \frac{d^{2} k}{(2 \pi)^{2}} \frac{1}{\left(k^{2}+m_{0}^{2}\right)\left((p+k)^{2}+m_{0}^{2}\right)} \\
\tilde{\pi}\left(p^{2}\right)=2 \int \frac{d^{2} k}{(2 \pi)^{2}} \frac{(k \cdot(k+p))^{2}}{\left(k^{2}+m_{0}^{2}\right)\left((p+k)^{2}+m_{0}^{2}\right)} .
\end{gathered}
$$

It is obvious from (14c) that the $\eta$ propagator has quadratic and logarthimic divergences and therefore needs regularization before attempting to compute the self energy. Eqs.(14) can be exactly computed and one finds :

$$
\begin{gathered}
\pi\left(p^{2}\right)=\frac{1}{2 \pi p^{2} \xi} \ln \frac{\xi+1}{\xi-1} \\
\tilde{\pi}\left(p^{2}\right)=\pi^{*}\left(p^{2}\right)+\frac{\Lambda^{2}}{2 \pi}-\frac{1}{4 \pi}\left(p^{2}+4 m_{0}^{2}\right) \ln \frac{\Lambda^{2}}{m_{0}^{2}}
\end{gathered}
$$

where the finite regularized propagator is :

$$
\pi^{*}\left(p^{2}\right)=2 m_{0}^{4} \pi\left(p^{2}\right)+\frac{p^{2}}{4 \pi}\left(\xi \ln \frac{\xi+1}{\xi-1}-1\right)
$$

where

$$
\xi=\left(1+\frac{4 m_{0}^{2}}{p^{2}}\right)^{\frac{1}{2}}
$$

The self energy $\Sigma$ can be computed from the diagrams of Fig(2). These dia- 
grams are of order $\frac{1}{D}$ and represent the quantum fluctuations:

$$
\begin{aligned}
\Sigma(p)= & \int \frac{d^{2} k}{(2 \pi)^{2}} \frac{\pi^{-1}\left(k^{2}\right)}{\left((p+k)^{2}+m_{0}^{2}\right)}+\frac{1}{2} \int \frac{d^{2} k}{(2 \pi)^{2}} \frac{\pi^{*-1}\left(k^{2}\right)(k \cdot(k+p))^{2}}{\left((p+k)^{2}+m_{0}^{2}\right)} \\
& -\int \frac{d^{2} k}{(2 \pi)^{2}} \int \frac{d^{2} q}{(2 \pi)^{2}} \frac{\pi^{-1}(0)}{\left(q^{2}+m_{0}^{2}\right)^{2}} \frac{\pi^{-1}\left(k^{2}\right)}{\left((q+k)^{2}+m_{0}^{2}\right)} \\
- & \frac{1}{2} \int \frac{d^{2} k}{(2 \pi)^{2}} \int \frac{d^{2} q}{(2 \pi)^{2}} \frac{\pi^{-1}(0)}{\left(q^{2}+m_{0}^{2}\right)^{2}} \frac{\pi^{*-1}\left(k^{2}\right)(k \cdot(k+q))^{2}}{\left((q+k)^{2}+m_{0}^{2}\right)} .
\end{aligned}
$$

A Taylor expansion of the self energy about zero momentum leads to mass and wave function renormalizations and a remaining piece $\tilde{\Sigma}$ which must be finite for the theory to be renormalizable. The propagator now reads:

$$
\frac{Z}{\left(p^{2}+m^{2}+\frac{1}{D} \tilde{\Sigma}_{\text {finite }}(p)\right)} \text {. }
$$

where

$$
Z=1-\frac{1}{D} \Sigma^{\prime}(0)
$$

is the wave function renormalization and

$$
m^{2}=m_{0}^{2}+\frac{1}{D}\left(\Sigma(0)-m_{0}^{2} \Sigma^{\prime}(0)\right)
$$

is mass renormalization. In order to calculate the finite regularized self energy we need to simplify further Eq.(16). Using (14) we can replace zero-momentum insertions of the $\sigma$ and $\rho$ fields and rewrite (16) as:

$$
\begin{gathered}
\Sigma(p)=\Sigma_{1}(p)+\int \frac{d^{2} k}{(2 \pi)^{2}} \frac{\pi^{-1}\left(k^{2}\right)}{\left((p+k)^{2}+m_{0}^{2}\right)}+\frac{1}{2} \pi(0)^{-1} \int \frac{d^{2} k}{(2 \pi)^{2}} \pi^{-1}\left(k^{2}\right) \frac{\partial}{m_{0}^{2}} \pi\left(k^{2}\right) \\
+\frac{1}{2} \int \frac{d^{2} k}{(2 \pi)^{2}} \frac{\pi^{*-1}\left(k^{2}\right)(k \cdot(k+p))^{2}}{\left((p+k)^{2}+m_{0}^{2}\right)}+\frac{1}{4} \pi(0)^{-1} \int \frac{d^{2} k}{(2 \pi)^{2}} \pi^{*-1}\left(k^{2}\right) \frac{\partial}{m_{0}^{2}} \pi^{*}\left(k^{2}\right) \\
+\frac{1}{2} m_{0}^{2} \log \frac{\Lambda^{2}}{m_{0}^{2}} \int \frac{d^{2} k}{(2 \pi)^{2}} \pi^{*-1}\left(k^{2}\right)-\frac{1}{2} \int \frac{d^{2} k}{(2 \pi)^{2}} k^{2} \pi^{*-1}\left(k^{2}\right)
\end{gathered}
$$

where $\Sigma_{1}(p)$ is a finite piece of the self energy. From the explicit form of the 
propagators in (15) one can derive the following identities:

$$
\begin{gathered}
\frac{\partial}{m_{0}^{2}} \pi\left(k^{2}\right)=\frac{-2}{k^{2} \xi^{2}}\left(\pi\left(k^{2}\right)+\pi(0)\right) \\
\frac{\partial}{m_{0}^{2}} \pi^{*}\left(k^{2}\right)=\left(\frac{1}{m_{0}^{2}}-\frac{2}{k^{2} \xi^{2}}\right) \pi^{*}\left(k^{2}\right)-\frac{2}{k^{2} \xi^{2}} \pi^{*}(0)+2 m_{0}^{2} \pi\left(k^{2}\right)-\frac{k^{2}}{4 \pi m_{0}^{2} \xi} \ln \frac{\xi+1}{\xi-1}-\frac{1}{2 \pi \xi^{2}} .
\end{gathered}
$$

Inserting (20) into (19) we obtain:

$$
\begin{gathered}
\Sigma(p)=\Sigma_{1}(p)+\frac{\Lambda^{2}}{4}-\frac{3}{2} m_{0}^{2} \log \frac{\Lambda^{2}}{4 m_{0}^{2}}+\int \frac{d^{2} k}{(2 \pi)^{2}} \frac{\pi^{-1}\left(k^{2}\right)}{\left((p+k)^{2}+m_{0}^{2}\right)}-\int \frac{d^{2} k}{(2 \pi)^{2}} \frac{\pi^{-1}\left(k^{2}\right)}{\left(k^{2}+4 m_{0}^{2}\right)} \\
+\frac{1}{2} \int \frac{d^{2} k}{(2 \pi)^{2}} \frac{\pi^{*-1}\left(k^{2}\right)(k \cdot(k+p))^{2}}{\left((p+k)^{2}+m_{0}^{2}\right)}-\frac{1}{2} \int \frac{d^{2} k}{(2 \pi)^{2}} k^{2} \pi^{*-1}\left(k^{2}\right)-\frac{m_{0}^{2}}{2} \int \frac{d^{2} k}{(2 \pi)^{2}} \pi^{*-1}\left(k^{2}\right) \\
+\frac{1}{2} m_{0}^{2} \log \frac{\Lambda^{2}}{m_{0}^{2}} \int \frac{d^{2} k}{(2 \pi)^{2}} \pi^{*-1}\left(k^{2}\right)+m_{0}^{4} \int \frac{d^{2} k}{(2 \pi)^{2}} \frac{\pi^{*-1}\left(k^{2}\right)}{\left(k^{2}+4 m_{0}^{2}\right)} \\
+2 \pi m_{0}^{4} \int \frac{d^{2} k}{(2 \pi)^{2}} \pi^{*-1}\left(k^{2}\right) \pi\left(k^{2}\right)-\frac{1}{4} \int \frac{d^{2} k}{(2 \pi)^{2}} \pi^{*-1}\left(k^{2}\right) \frac{k^{2}}{\xi} \ln \frac{\xi+1}{\xi-1} .
\end{gathered}
$$

Now we are in a good position to regularize (21). We proceed by applying the SM regularization scheme [4] where one subtracts the highest powers of the integration variable appearing in the Taylor expansion of the integrands in (21). The divergences in (21) occur at the zeroth and first order expansion. Using the asymptotic limits of the propagators (15):

$$
\begin{gathered}
\pi_{a s y}\left(p^{2}\right)=\frac{1}{2 \pi p^{2}} \log \frac{p^{2}}{m_{0}^{2}} \\
\pi_{a s y}^{*}\left(p^{2}\right)=\frac{p^{2}}{4 \pi} \log \frac{p^{2}}{m_{0}^{2}}
\end{gathered}
$$

we obtain the following finite regularized self energy:

$$
\Sigma_{\text {finite }}(p)=\Sigma(p)+m_{0}^{2} \log \frac{\Lambda^{2}}{m_{0}^{2}}\left(1-\frac{1}{2} I_{0}\right)-\frac{m_{0}^{2}}{4} I_{0}-\frac{1}{4}\left(p^{2}+m_{0}^{2}\right) I_{0}
$$


where

$$
I_{0}\left(\frac{\Lambda^{2}}{m_{0}^{2}}\right)=\int \frac{d^{2} k}{(2 \pi)^{2}} \frac{4 \pi}{k^{2} \log \frac{k^{2}}{m_{0}^{2}}}=\log \log \frac{\Lambda^{2}}{m_{0}^{2}}
$$

We have arranged $\Sigma_{\text {finite }}(p)$ in the above form so as it is easy to see how the above result (23) can be generalized to the interacting theory of rigid string with the long range Kalb-Ramond interactions presented in part II. We can now read from (23) the mass and wave function renormalizations:

$$
\begin{gathered}
m^{2}=m_{0}^{2}\left[1-\frac{1}{D}\left(\log \frac{\Lambda^{2}}{m_{0}^{2}}\left(1-\frac{1}{2} I_{0}\right)-\frac{1}{4} I_{0}\right)\right] \\
Z=1-\frac{1}{4 D} I_{0} .
\end{gathered}
$$

To obtain the beta function we hold $m^{2}\left(\frac{\Lambda^{2}}{m_{0}^{2}}, t\right)$ fixed we obtain:

$$
\begin{gathered}
\beta(t)=-\left(\frac{\partial \log m^{2}}{\partial t}\right)^{-1} \\
\beta\left(t^{*}\right)=-t^{* 2}\left(1-\frac{1}{2 D}\right)\left(1+\frac{1}{2 D}\left(\frac{1}{2} t^{*}-\log t^{*}\right)\right)
\end{gathered}
$$

where we have used the mass gap equation (10a) and defined $t^{*}=\frac{D t}{8 \pi}$. Eq.(25) is the main result of this letter. It shows that the free rigid string theory is asymptotically free to the sub-leading quantum corrections in the large D limit. The critical point is still the ultra violet fixed point $t=t_{c}=0$. Therefore at large distance scales in the disordered phase $t>t_{c}$ the extrinsic curvature of the string becomes irrelevant and the resulting classical limit is the usual Nambu-Goto string which is free of ghosts. The rigid string is a higher derivative theory and therefore can have the typical pathologies, namely classical runaway solutions and ghosts appearing in the perturbative propagator. However the non-perturbative (in the coupling t) result (25) shows that the regulated quantum theory is consistent. the extrinsic curvature term only survives at very short distance scales and does not affect the poles of the propagator, which is a large distance property. Indeed in [2], and [5] we have proved that rigid QED which is a higher derivative theory is a consistent quantum theory both in the leading and sub-leading orders in large $\mathrm{N}$ limit and that the phase transition survives sub-leading quantum fluctuations. 


\section{Acknowledgement}

I am very grateful to Prof. A. Polyakov for his constant encouragement and extensive support over the last year and a half and for suggesting that we address the quantum stability of the phase transition both in the model of rigid QED and that of rigid strings coupled to long range interactions [6]. I am also grateful to Prof. Y. Nambu for his extensive support, encouragement and long discussions over the last two years without which we could not have gone far in our investigations. I also thank P.Ramond, C. Thorn, and Z. Qui for constructive discussions and suggestions. Finally my gratitude to my friend D. Zoller for a fruitful and devoted collaboration over the years.

\section{References}

[1] A. Polyakov, Nucl. Phys. B268 (1986) 406 ; A. Polyakov, Gauge fields, and Strings, Vol.3, harwood academic publishers

[2] M. Awada and D. Zoller, Phys.Lett B325 (1994) 119

[3] M. Awada and D. Zoller, Phys.Lett B325 (1994) 115

[4] A. Polyakov, Gauge fields, and Strings, Vol.3, harwood academic publishers, J.Orloff and R.Brout, Nucl. Phys. B270 [FS16],273 (1986), M. Campostrini and P.Rossi, Phys. Rev.D 45, 618 (1992) ; 46, 2741 (1992), H. Flyvberg, Nucl. Phys. B 348, 714, (1991).

[5] M. Awada, D. Zoller, and J. Clark, Cincinnati preprint June -1 -(1994) 


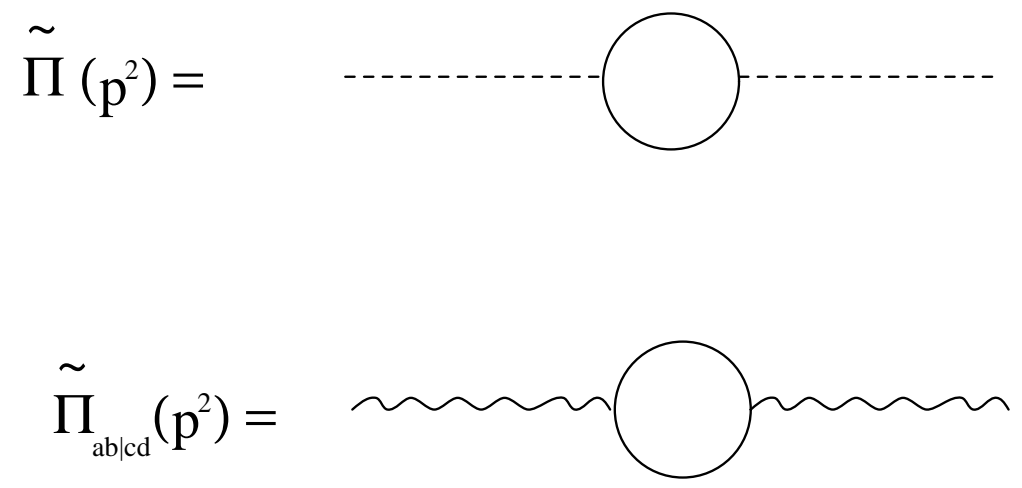

Fig(1)

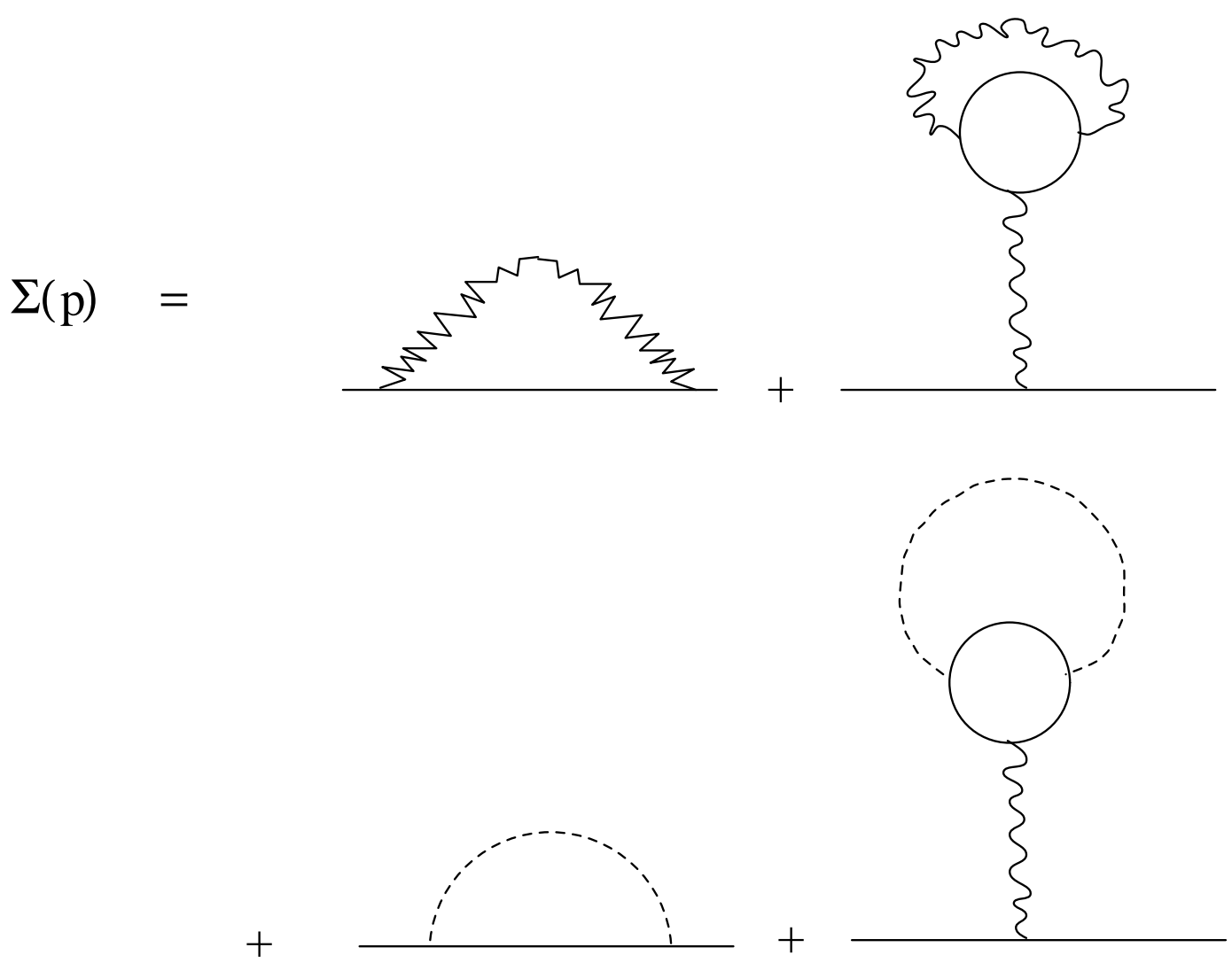

Fig(2) 